\title{
Macrolides antiparasitaires : propriétés pharmacologiques générales et recommandations d'usage dans le contexte vétérinaire africain
}

\author{
T. Bengone-Ndong ${ }^{1}$ M. Alvinerie ${ }^{2 *}$
}

Mots-clés

Parasitisme - Médicament vétérinaire - Propriété pharmacologique - Efficacité - Afrique.

\begin{abstract}
Résumé
Les macrolides antiparasitaires, ou endectocides, représentent la classe d'antiparasitaires la plus récente. Cette classe est homogène en raison du mode d'action unique et spécifique, glutaminergique, et de ses propriétés pharmacologiques. Ces substances ont un spectre d'activité étendue à de nombreux nématodes ainsi qu'à de nombreux insectes et acariens et, du fait de leur structure et des formulations galéniques associées, présentent également une importante rémanence. Les endectocides sont malheureusement commercialisés sur le marché africain le plus souvent sans aucune procédure d'enregistrement. De plus, l'introduction des formulations génériques a facilité un usage massif et répétitif susceptible de contribuer à des échecs thérapeutiques. II convient de donner à ces antiparasitaires les meilleures conditions pour exercer leur action en milieu tropical africain, en prenant en compte notamment les particularités des races locales et les contraintes environnementales. Parmi les moyens à mettre en œuvre, la sélection de la voie d'administration la plus performante constitue le premier élément à prendre en compte. De même, les différences observées entre les espèces animales cibles ainsi que les particularismes physiologiques doivent conduire à une utilisation mieux adaptée.
\end{abstract}

\section{INTRODUCTION}

Le développement de l'élevage dans les pays d'Afrique de l'Ouest et du Centre est limité par des contraintes alimentaires et sanitaires, et la lutte contre les maladies parasitaires reste l'un des défis à relever. La part des antiparasitaires avoisine déjà 50 p. 100 des médicaments vétérinaires dans la plupart de ces pays (2). Les macrolides endectocides constituent dans ce cadre la classe thérapeutique la plus largement utilisée en raison tant de leur spectre d'action que de leur efficacité remarquable. Comme tous les médicaments vétérinaires, ces endectocides sont généralement

1. Ecole inter-Etats des sciences et médecine vétérinaires, BP 5077, Dakar Fann, Sénégal

2. Laboratoire de pharmacologie - toxicologie, Inra, 180 chemin de Tournefeuille, BP 3, 31931 Toulouse Cedex 09, France

* Auteur pour la correspondance

Tél. : +33 (0)561285137; fax : +33 (0)5 61285310

E-mail : michel.Alvinerie@toulouse.inra.fr commercialisés sur le marché africain après une procédure sommaire d'enregistrement dans le meilleur des cas.

Des actions doivent être menées dans ces pays pour sensibiliser les responsables sur la nécessité d'accorder un intérêt particulier à la qualité et à l'efficacité des macrolides endectocides importés et dont les études sont réalisées sur des espèces occidentales. Les actions à mener sont d'autant plus indispensables que de nombreux problèmes (résistance) associés à la défaillance de cette famille thérapeutique commencent à émerger à travers le monde et proviennent essentiellement de leur emploi excessif et de choix de traitement erronés. Il convient de donner aux antiparasitaires endectocides les meilleures conditions pour exercer leur action en milieu tropical africain en prenant en compte notamment les particularités des races locales et les contraintes environnementales.

Dans cet article, les auteurs ont réalisé une synthèse de données générales sur les macrolides endectocides. Par la suite, est proposée une analyse des facteurs impliqués dans l'efficacité de ces endectocides pouvant faire l'objet d'une évaluation pharmacocinétique. L'intérêt de l'outil pharmacocinétique repose sur le fait 
(généralement admis) que l'activité anthelminthique des endectocides est liée en partie à la présence effective du principe actif sur le site d'action en termes de concentration et de durée. Cette analyse a amené les auteurs à formuler des recommandations pour une meilleure utilisation des antiparasitaires endectocides dans le contexte vétérinaire africain.

\section{- PROPRIETES GENERALES DES MACROLIDES ENDECTOCIDES}

\section{Historique}

Les recherches sur les antiparasitaires ont été orientées, à partir de 1975, vers des substances d'origine naturelle novatrices, radicalement différentes de celles qui étaient alors utilisées en thérapeutique vétérinaire. Ces recherches ont conduit à un vaste programme de criblage aléatoire des microorganismes telluriques collectés dans le monde entier et analysés en laboratoire.

En 1979, les premiers résultats ont été obtenus grâce à un bouillon de fermentation provenant d'un échantillon de sol collecté à Kawana (Ito City, Japon) par l'institut Kitasato. Celui-ci montra une activité antiparasitaire remarquable dans un test in vivo sur des souris infestées par Nematospiroides dubius, un nématode dont certains isolats sont résistants aux anthelminthiques classiques, notamment aux benzimidazoles $(24,37,59)$. Un agent inconnu fut alors isolé et son fort potentiel fut mis en évidence par son activité naturelle dans des proportions infimes : $1 \mu \mathrm{g}$ par gramme de nourriture distribuée, soit $1 \mathrm{ppm}$ de la ration. Cette activité s'est révélée très supérieure à celle des autres anthelminthiques connus (24).

Le nom de cette nouvelle famille de composés découla de ses propriétés acaricide, insecticide et nématodicide : avermectines $(a$ : anti ; verm : ver ; ect : ectoparasite) (62). De cette capacité à éliminer les endoparasites (nématodes) et les ectoparasites (arthropodes) est apparu le concept nouveau d' « endectocide ».

Les milbémycines constituent le deuxième groupe d'endectocides. Ce sont des composés acaricides et insecticides qui ont été découverts en 1973 mais dont le potentiel anthelminthique n'a été réalisé que lors de l'avènement des avermectines (62).

Les avermectines et les milbémycines sont issues de la culture de bactéries filamenteuses du genre Streptomyces ; elles ont été introduites en thérapeutique vétérinaire en 1981 (ivermectine) et ont succédé à la phénothiazine, au thiabendazole et à divers autres benzimidazoles, en particulier chez les bovins. Leur activité s'exerce avec une dose faible $(0,2 \mathrm{mg} / \mathrm{kg})$ par comparaison avec la phénothiazine $(600 \mathrm{mg} / \mathrm{kg})$, le thiabendazole $(50 \mathrm{mg} / \mathrm{kg})$ et l'oxfendazole $(5 \mathrm{mg} / \mathrm{kg})$. Elles constituent à ce jour la classe thérapeutique la plus largement utilisée dans la lutte contre les parasites des bovins.

\section{Structure des avermectines et des milbémycines}

Les avermectines et les milbémycines présentent une même structure générale de lactone macrocyclique. La différence structurale majeure entre les deux groupes consiste au groupement disaccharidique (substituant bisoleandrosyloxy) sur le carbone numéro 13 des avermectines. Les milbémycines en sont dépourvues ; ce sont donc des avermectines déglycosylées (aglycones des avermectines) (figures 1 et 2).

\section{Avermectines}

Les avermectines (figure 1) sont issues de la culture de Streptomyces avermitilis. Huit composés naturels ont été isolés : $\mathrm{A}_{l a}, \mathrm{~A}_{1 b}$, $\mathrm{A}_{2 a}, \mathrm{~A}_{2 b}, \mathrm{~B}_{1 a}, \mathrm{~B}_{1 b}, \mathrm{~B}_{2 a}$ et $\mathrm{B}_{2 b}$. Les composés $\mathrm{A}$ possèdent une groupement méthoxyle sur le carbone numéro 5, alors que les

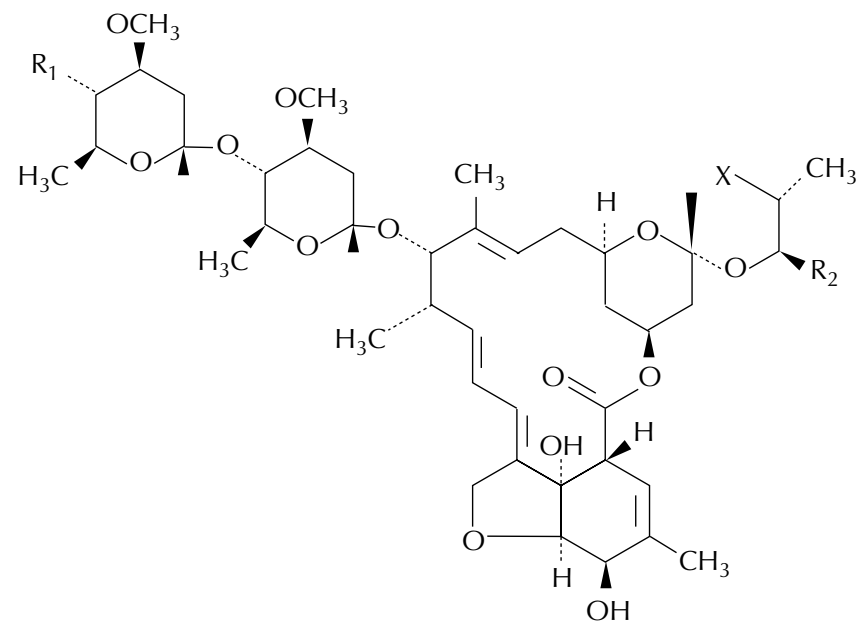

Figure 1 : structure chimique des principales avermectines. 1) Ivermectine : mélange de plus de $80 \%$ de $B_{1 a}$ et moins de $20 \%$ de $B_{1 b}$. Composant $B_{1 a}: R_{1}=O H ; R_{2}=$ $\mathrm{CH}\left(\mathrm{CH}_{3}\right) \mathrm{CH}_{2} \mathrm{CH}_{3} ; \mathrm{X}=-\mathrm{CH}_{2} \mathrm{CH}_{2}-$. Composant $\mathrm{B}_{1 b}: \mathrm{R}_{1}=$ $\mathrm{OH} ; \mathrm{R}_{2}=\mathrm{CH}\left(\mathrm{CH}_{3}\right)_{2} ; \mathrm{X}=-\mathrm{CH}_{2} \mathrm{CH}_{2}-$

2) Abamectine : mélange de plus de $80 \%$ de $B_{1 a}$ et moins de $20 \%$ de $B_{1 b}$. Composant $B_{1 a}: R_{1}=O H ; R_{2}=$ $\mathrm{CH}\left(\mathrm{CH}_{3}\right) \mathrm{CH}_{2} \mathrm{CH}_{3} ; \mathrm{X}=-\mathrm{CH}=\mathrm{CH}$-. Composant $\mathrm{B}_{1 b}: \mathrm{R}_{1}=$ $\mathrm{OH} ; \mathrm{R}_{2}=\mathrm{CH}\left(\mathrm{CH}_{3}\right)_{2} ; \mathrm{X}=-\mathrm{CH}=\mathrm{CH}$.

3) Eprinomectine : mélange de plus de $80 \%$ de $B_{1 a}$ et moins de $20 \%$ de $B_{1 b}$. Composant $B_{1 a}: R_{1}=N H_{C O C H} ; R_{2}=$ $\mathrm{CH}\left(\mathrm{CH}_{3}\right) \mathrm{CH}_{2} \mathrm{CH}_{3} ; \mathrm{X}=-\mathrm{CH}=\mathrm{CH}$ - Composant $\mathrm{B}_{1 b}: \mathrm{R}_{1}=$ $\mathrm{NHCOCH}_{3} ; \mathrm{R}_{2}=\mathrm{CH}\left(\mathrm{CH}_{3}\right)_{2} ; \mathrm{X}=-\mathrm{CH}=\mathrm{CH}$ -

4) Doramectine : $R_{1}=O H ; R_{2}=$ cyclohexyle; $X=$ $-\mathrm{CH}=\mathrm{CH}$.

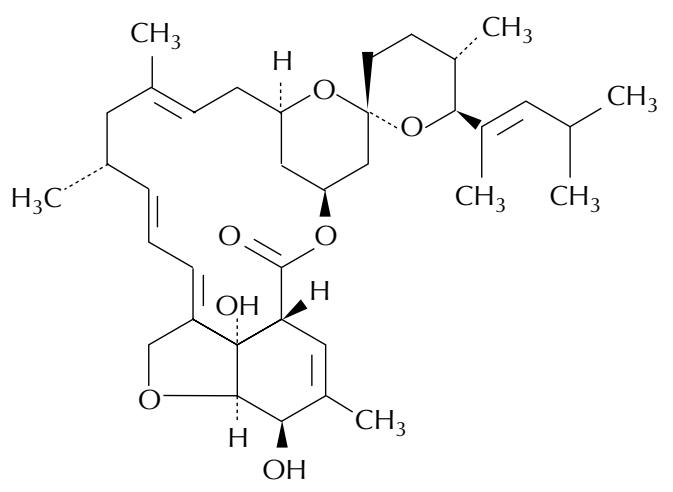

Figure 2 : structure chimique de la moxidectine (famille des milbémycines).

composés B portent une groupement hydroxyle. La liaison entre les atomes de carbone 22 et 23 est double dans le cas des composés 1 ; elle est simple dans la structure des composés 2 . Enfin, les composés $a$ possèdent un substituant butyle en position 25 alors qu'il s'agit d'un isopropyle dans le cas des composés $b$. Les homologues $a$ et $b$ ont une activité presque identique. Leur séparation au cours de la fermentation à grande échelle est difficile et sans intérêt, de sorte que, dans la littérature, l'on ne considère quelques fois que quatre types d'avermectines: $\mathrm{A}_{1}, \mathrm{~A}_{2}, \mathrm{~B}_{1}$ et $\mathrm{B}_{2}$.

L'ivermectine (22,23-dihydro-avermectine $\mathrm{B}_{1}$ ) a été la première avermectine commercialisée $(15,25)$. Elle est obtenue par hydrogénation sélective de la double liaison 22-23 de l'avermectine $\mathrm{B}_{l}$. $\mathrm{Au}$ plan structural, il s'agit d'un intermédiaire entre $\mathrm{B}_{1}$ et $\mathrm{B}_{2}$. Elle possède, au plan biologique, un excellent potentiel antiparasitaire 
(proche de $\mathrm{B}_{1}$ ), avec une sécurité d'emploi (s'approchant de $\mathrm{B}_{2}$ ). Sa $D L_{50}$ est estimée à $30 \mathrm{mg} / \mathrm{kg}$ chez la souris (15). Compte tenu de son ancienneté, elle possède le plus large spectre d'activité reconnu par les autorisations de mise sur le marché car de nombreux travaux ont été réalisés en vue d'explorer le maximum de cibles possibles.

L'abamectine (avermectine $\mathrm{B}_{l}$ ) est aussi un produit de fermentation de $S$. avermitilis. Sa production est plus simple que celle de l'ivermectine. Elle est plus active sur les nématodes que l'ivermectine mais un peu moins efficace sur quelques arthropodes, bien que son utilisation en protection des cultures soit en relation avec son activité acaricide et insecticide (24).

La doramectine (25-cyclohexyl-avermectine $\mathrm{B}_{1}$ ) est un produit de fermentation d'une souche mutante de $S$. avermitilis en présence de l'acide cyclohexanecarboxylique. Son spectre d'activité se rapproche de celui de l'avermectine $\mathrm{B}_{l}$, ce qui s'explique par leurs structures très proches qui ne diffèrent que par un radical cyclohexyle en position 25. Celui-ci serait très lipophile et ferait que la demi-vie tissulaire de la doramectine soit beaucoup plus longue (29).

L'éprinomectine [4'-(épiacétylamino)-4'-désoxy-avermectine $\mathrm{B}_{1}$ ] est issue de la fermentation $S$. avermitilis, comme l'ivermectine et l'abamectine. Elle a été sélectionnée parmi plus de 500 lactones macrocycliques en vue d'obtenir le spectre et la marge de sécurité les plus larges avec, en particulier, les concentrations les plus faibles dans le lait, permettant ainsi son emploi chez les vaches laitières en production $(7,64,65)$.

\section{Milbémycines}

Les milbémycines naturelles (figure 2) sont issues de la fermentation de Streptomyces hygroscopicus et Streptomyces cyaneogriseus. On distingue également dans ce groupe des composés $A$ (groupement méthoxyle en position 5) et des composés $B$ (groupement hydroxyle en position 5). La liaison entre les atomes de carbone 22 et 23 est toujours une liaison simple, comme dans le cas des avermectines 2 . Le carbone 25 porte un groupement méthyle, éthyle ou une chaîne latérale substituée $(14,66)$.

La moxidectine (dérivé méthoxime de la milbémycine) contient un cycle lactonique semi-synthétique. Son mode d'action est comparable à celui de l'ivermectine, mais des différences existent entre les deux molécules. L'effet rémanent est très nettement plus long pour la moxidectine que pour l'ivermectine. La moxidectine n'est pas toxique pour les chiens de race Colley; elle est active sur les Chorioptes et permet donc la guérison des bovins atteints par cet acarien ; elle n'a pas d'action sur les microfilaires chez le chien contrairement à l'ivermectine. Ces particularités montrent qu'il s'agit bien d'un produit différent tout en ayant des points communs avec les avermectines.

\section{Relation «structure-activité »}

Toutes les avermectines et les milbémycines ont un large spectre d'action, mais il existe des variations d'une série à une autre, ce qui indique que l'activité est en étroite relation avec la structure. Des huit composés naturels obtenus à partir de Streptomyces avermitilis, seulement trois sont produits en quantité lors de la fermentation : $\mathrm{A}_{2 a}, \mathrm{~B}_{1 a}$ et $\mathrm{B}_{2 a}$; ils ont fait l'objet de nombreux travaux. Des études des relations entre la structure et l'activité des différentes molécules ont montré que l'homologue $B_{1}$ possède le meilleur potentiel et spectre d'action contre les nématodes, alors que $\mathrm{B}_{2}$ présente une meilleure sécurité d'emploi (tableau I). Chez la souris, la $D L_{50}$ du composé $\mathrm{B}_{1}$ est estimé à $15 \mathrm{mg} / \mathrm{kg}$ alors que celle de $\mathrm{B}_{2}$ est de 30 $\mathrm{mg} / \mathrm{kg}$ (63). On comprend dès lors que la plupart des avermectines et milbémycines disponibles sur le marché du médicament aient été développées à partir de ces deux homologues.

\section{Tableau I}

Efficacité des avermectines naturelles contre six espèces de nématodes chez le mouton (Shoop et coll., 1995, Vet. Parasitol.)

$\begin{array}{lccccccc}\text { Structure } & \begin{array}{c}\text { Dose } \\ (\mathbf{m g} / \mathbf{k g})\end{array} & \text { Hc } & \text { Oc } & \text { Ta } & \text { Tc } & \text { Csp } & \text { Oec } \\ \text { Avermectine } A_{1} & 0,1 & 2 & 2 & 0 & 0 & 2 & 0 \\ \text { Avermectine } A_{2} & 0,1 & 3 & 3 & 3 & 3 & 0 & 3 \\ \text { Avermectine } B_{1} & 0,1 & 3 & 3 & 3 & 3 & 3 & 3 \\ \text { Avermectine } B_{2} & 0,1 & 3 & 3 & 3 & 3 & 2 & 3\end{array}$

Hc : Haemonchus contortus ; Oc : Teladorsagia circumcincta;

Ta : Trichostrongylus axei; Tc: Trichostrongylus colubriformis ;

Csp : Cooperia spp.; Oec : Oesophagostomum columbianum.

Code efficacité

$0:<50 \% ; 1: 50-74 \% ; 2: 75-90 \% ; 3:>90 \%$

\section{Spécialités vétérinaires disponibles}

Le succès de l'ivermectine en thérapeutique a engendré le développement des études sur la relation structure-activité des endectocides. Les essais sur des analogues structuraux se sont multipliés et ont eu pour but la recherche de la molécule la plus performante. Chaque composé provenant d'une modification structurale des molécules parentales est original de par son spectre et sa sécurité d'emploi (37).

Cinq principaux macrolides endectocides sont actuellement présents sur le marché des médicaments vétérinaires : l'ivermectine, l'abamectine, la doramectine, l'éprinomectine et la moxidectine. Ils sont constitués le plus souvent de mélanges racémiques de molécules parentales naturelles ou de synthèse (figure 1). Ces molécules présentent la faculté d'être facilement associées à plusieurs formes galéniques : préparations orales, formes injectables, formes orales, pour-on, diffuseurs. L'association des propriétés intrinsèques des macrolides antiparasitaires à celle des excipients permet l'obtention d'une action persistante. Celle-ci assure une extension du spectre d'activité et une protection des animaux visà-vis des réinfestations (tableau II). Les formulations galéniques ne présentent pas toutes les mêmes indications antiparasitaires et ne sont pas toutes destinées aux mêmes animaux. Il est donc nécessaire, pour envisager l'utilisation des macrolides antiparasitaires, de distinguer les animaux traités et les formulations employées.

Les doses administrées dépendent des espèces cibles et de la voie d'administration (tableau III). D'une façon générale, la posologie des endectocides dans leur forme injectable est de $0,2 \mathrm{mg} / \mathrm{kg}$ et de $0,5 \mathrm{mg} / \mathrm{kg}$ pour les formulations pour-on chez les herbivores. Le bolus intraruminal d'ivermectine utilise $8,6 \mathrm{mg} / \mathrm{kg}$. Ces quantités permettent d'assurer un large spectre sur les nématodes chez les espèces animales cibles.

\section{Mode d'action}

L'action des endectocides est lente et reliée à la fois à l'action intrinsèque du médicament sur le parasite cible et à la présence de concentrations significatives en termes de niveau et de durée sur le site d'action. De nombreuses études ont permis d'établir un mode d'action unique pour l'ensemble des macrolides endectocides. Ce mode d'action fait intervenir le système glutaminergique.

Les macrolides endectocides agissent sur la transmission nerveuse. Ils se fixent sur un récepteur au glutamate, au niveau des canaux 


\section{Tableau II}

Exemple de rémanence d'activité des endectocides

\begin{tabular}{|c|c|c|c|}
\hline \multirow[t]{2}{*}{ Molécule } & \multicolumn{2}{|c|}{ Rémanence d'activité } & \multirow[t]{2}{*}{ Référence } \\
\hline & Ostertagia ostertagi & Dictyocaulus viviparus & \\
\hline Abamectine & 10-14 jours & 21 jours & Dobson et coll., 2001, Aust. vet. J. \\
\hline Ivermectine & 7-14 jours & 14-21 jours & $\begin{array}{l}\text { Williams et Broussard, 1995, Am. J. vet. Res. ; Williams et } \\
\text { coll., 1999, Vet. Parasitol. }\end{array}$ \\
\hline Doramectine & 21-28 jours & $>28$ jours & $\begin{array}{l}\text { Pitt et coll., 1997, Vet. Parasitol. ; Vercruysse et coll., 1998, } \\
\text { Vet. Rec. ; Wardhaugh et coll., 2001, Aust. vet. J; } \\
\text { Williams et coll., 1999, Vet. Parasitol. }\end{array}$ \\
\hline Eprinomectine & 28 jours & 28 jours & $\begin{array}{l}\text { Pitt et coll., 1997, Vet. Parasitol. ; Vercruysse et coll., 1998, } \\
\text { Vet. Rec. ; Wardhaugh et coll., 2001, Aust. vet. J; } \\
\text { Williams et coll., 1999, Vet. Parasitol. }\end{array}$ \\
\hline Moxidectine & 35 jours & 35 jours & $\begin{array}{l}\text { Abbott et coll., 1995, Aust. vet. J. ; Kerbœuf et coll., 1995, } \\
\text { Vet. Rec. ; Wardhaugh et coll., 2001, Aust. vet. J. ; } \\
\text { Williams et Broussard, 1995, Am. J. vet. Res. ; } \\
\text { Williams et coll., 1999, Vet. Parasitol. }\end{array}$ \\
\hline
\end{tabular}

Tableau III

Principaux antiparasitaires endectocides utilisés en médecine vétérinaire

chlore, à proximité d'un récepteur Gaba (acide gamma amino butyrique) et d'un récepteur aux benzodiapézines. Cette fixation provoque un blocage des canaux chlore en position ouverte et donc un flux entrant d'ions chlore au sein des cellules nerveuses des parasites (la glutamase n'ayant aucune action sur les avermectines et milbémycines). Il s'ensuit une hyperpolarisation cellulaire qui bloque toute activité nerveuse et entraîne une paralysie flasque ( 9 , $13,35,38)$. Une action semblable est obtenue avec un neuromédiateur inhibiteur Gaba. Les macrolides endectocides ont ainsi été considérés comme ayant un effet Gaba-ergique (libération accrue de Gaba associée peut-être à une potentialisation de son action). Mais leur effet pourrait être en fait Gaba-mimétique (68).

Ce système neuromédiateur faisant intervenir différents récepteurs dont celui du Gaba est très important chez les invertébrés, arthropodes et nématodes, montrant ainsi le spectre d'activité des macrolides endectocides. Les structures concernées sont retrouvées au niveau de la jonction interneuronale chez les nématodes et au niveau de la jonction neuromusculaire chez les arthropodes. L'action des endectocides se manifeste donc par une inhibition de l'activité électrique des cellules nerveuses des nématodes et des cellules musculaires des arthropodes, entraînant une paralysie flasque et irréversible $(9,13,35,38)$.

Ces molécules sont en revanche inactives sur les plathelminthes (trématodes et cestodes). Les vers plats ont en effet un système nerveux moins développé et ne possèdent pas de récepteurs au glutamate similaires à ceux des nématodes et des arthropodes sur lesquels se fixent les macrolides antiparasitaires $(18,42,62,63)$. Chez les mammifères, ce type de récepteurs ne sont présents qu'au niveau du système nerveux central et sont protégés par la multidrug-resistance-Pglycoprotéine (MDR-P-glycoprotéine). La MDR-P-glycoprotéine est une protéine membranaire présente dans de nombreuses cellules de l'organisme qui, par un mécanisme de capture et d'excrétion active, participe au maintien de l'intégrité de l'organisme vis-à-vis des xénobiotiques ; elle est majoritairement présente au niveau de la barrière hémato-encéphalique $(61,77)$. En cas de dysfonctionnement de la MDR-P-glycoprotéine, l'accès aux récepteurs Gaba n'est plus protégé, ce qui pourrait être à l'origine d'une toxicité accrue pour l'organisme. Il a également été montré que la sensibilité particulière du chien Colley à l'ivermectine était liée à une mutation sur le gène $\mathrm{MDR}_{1}$ codant pour une MDR-glycoprotéine. Il est donc possible de 
détecter, par un test génétique, les animaux porteurs de cette mutation et donc sensibles à l'ivermectine (60).

En plus de leur effet majoritairement paralysant, les endectocides peuvent avoir des effets sur la reproduction et le cycle des parasites. On peut noter dans le cas de l'ivermectine une action relativement délétère sur les fonctions de reproduction des tiques : inhibition de la ponte pour les femelles adultes et de la mue pour les stades nymphaux. Une inhibition des récepteurs au glutamate des muscles des organes reproducteurs femelles a été signalée chez Ascaris suum (27).

\section{Spectre d'activité}

Les lactones macrocycliques présentent une grande efficacité à l'égard de nombreux parasites internes et externes des animaux domestiques et leur activité s'exerce à de nombreux stades parasitaires. Elle comprend une action anthelminthique et une action insecticide acaricide.

\section{Action sur les nématodes digestifs et respiratoires}

Les endectocides sont actifs sur la majorité des nématodes parasites à localisation digestive et respiratoire. Ils sont particulièrement indiqués contre les strongles digestifs (Haemonchus, Ostertagia, Teladorsagia, Trichostrongylus, Cooperia, Nematodirus, Bunostomum, Oesophagostomum, Trichuris), les strongles respiratoires (Dictyocaulus, Protostrongylus, Muellerius), les anguillules (Strongyloides) et les trichures, chez les ruminants. Parmi ces nématodes, certains constituent des espèces limitantes car naturellement moins sensibles que d'autres. Il s'agit des Cooperia spp. et de Nematodirus spp. $(12,57,75)$.

Les endectocides ont une action adulticide, larvicide et, dans certains cas, sont actifs sur les larves de strongles entrant en hypobiose, notamment celles du genre Teladorsagia. Il existe des niveaux de sensibilité différents selon la nature du parasite.

Ces molécules sont inactives sur les vers plats (trématodes et cestodes), en raison de leur mode d'action, mais présentent une activité sur les souches de nématodes résistantes aux benzimidazoles.

\section{Action sur les parasites externes}

Les macrolides endectocides sont utilisés dans le traitement des ectoparasitoses des ruminants. Les formulations injectables sont actives sur les poux piqueurs (Haematopinus, Linognathus et Solenopotes), les mélanophages (Melaphagus ovinus) et les agents de gales du genre Sarcoptes ou Psoroptes $(34,39,65)$. Les pour-on, avec une distribution à la fois systémique et superficielle, ont un spectre d'activité élargi aux poux broyeurs (Bovicola syn. Damalinia) et aux agents de gales du genre Chorioptes. Une seule administration est généralement suffisante chez les bovins pour contrôler une phtiriose ou une gale. Les présentations orales sont insuffisamment actives sur les parasites externes et ne sont employées que pour une vermifugation des animaux.

\section{Distribution et élimination dans le lait}

Les endectocides sont fortement lipophiles ; ils sont distribués dans tous les tissus. Le stockage dans le tissu adipeux est particulièrement important ; ce dernier agit comme un réservoir ; il est à l'origine de la longue rémanence dans l'organisme. Leur métabolisation est faible et la quasi-totalité de la dose atteignant la circulation générale est éliminée dans les matières fécales sous forme du principe actif inchangé.

L'excrétion dans le lait constitue une caractéristique majeure de ces composés (l'ivermectine, la moxidectine et la doramectine sont éliminées dans le lait). La concentration de l'ivermectine dans le lait est équivalente à la concentration plasmatique et, au total, 5 p. 100 (chez la vache) et 15 p. 100 (chez la chèvre) de la dose totale administrée sont excrétés dans le lait. Cette importante élimination dans le lait justifie l'interdiction de leur usage chez les femelles en production lactée.

L'éprinomectine présente une élimination bien plus faible dans le lait ; le rapport lait/plasma est de 0,1 chez la vache et cette élimination ne met en jeu que 0,1 p. 100 de la dose totale administrée (7). Cette particularité autorise l'obtention d'un délai d'attente nul pour le lait.

\section{FACTEURS MODULANT L'EFFICACITE} DES ENDECTOCIDES

\section{Relation entre la pharmacocinétique et l'efficacité des endectocides}

La pharmacocinétique envisage l'action de l'organisme sur le médicament et son rôle dans l'estimation de l'efficacité des endectocides repose sur l'hypothèse selon laquelle le profil plasmatique reflète le profil de concentration du principe actif au niveau du site d'action. Il est de fait généralement admis que l'effet antiparasitaire d'un médicament est plus étroitement lié à la concentration plasmatique (mesurée par l'aire sous la courbe temps versus concentration) qu'à la dose administrée.

De récents travaux montrent l'étroite corrélation qui relie les concentrations plasmatiques aux concentrations observées au niveau de sites d'action tels que la peau ou le mucus abomasal (49). En effet, non seulement les profils d'évolution sont parallèles, mais, de plus, les concentrations dans les tissus cibles sont supérieures à celles observées dans le plasma (figure 3).

A titre d'exemple, chez les bovins, après administration souscutanée de moxidectine à la dose thérapeutique de $0,2 \mathrm{mg} / \mathrm{kg}$, les valeurs des AUC sont respectivement de $159 \mathrm{ng} / \mathrm{j} / \mathrm{ml}$ et $371 \mathrm{ng} / \mathrm{j} / \mathrm{ml}$ pour le plasma et le mucus abomasal, ce qui traduit un taux d'exposition deux fois supérieur pour le mucus. En revanche, le temps moyen de résidence (Mrt) présente une valeur identique dans les deux compartiments (8,60 et 8,90 jours), ce qui reflète une disposition cinétique parallèle.

Des résultats similaires ont été obtenus pour la doramectine et l'ivermectine (50). Dès lors, on peut considérer que, dans le cas des endectocides, les paramètres plasmatiques sont pertinents pour prédire l'activité anthelminthique au niveau du site d'action.

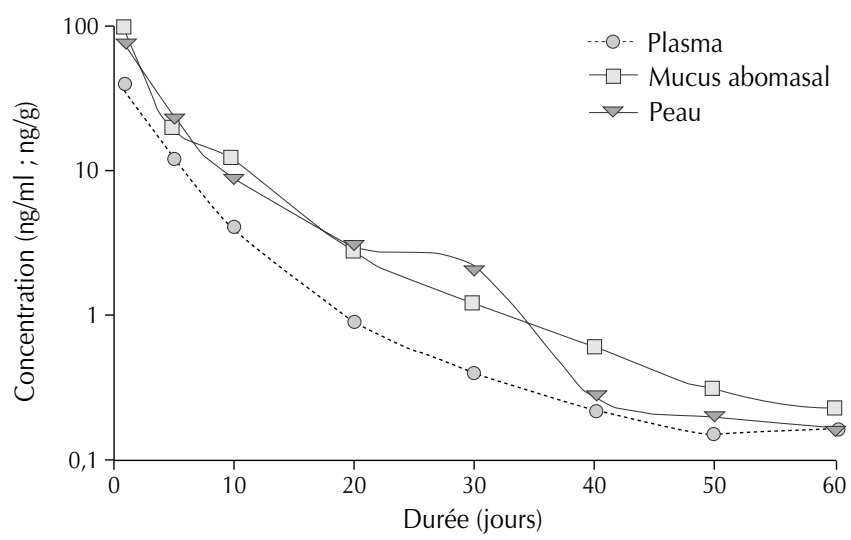

Figure 3 : relation entre les concentrations plasmatiques et les concentrations au niveau des tissus cibles après administration sous-cutanée de moxidectine à la dose de $0,2 \mathrm{mg} / \mathrm{kg} \mathrm{chez}$ les bovins (Lifschitz et coll., 1999, J. vet. Pharmacol. Ther.). 


\section{Influence du principe actif et de sa formulation}

Une comparaison du profil pharmacocinétique des trois endectocides majeurs (ivermectine, doramectine, moxidectine) administrés par voie sous-cutanée, dans le cadre d'une étude standardisée, a révélé un profil similaire pour l'ivermectine et la doramectine tandis que la moxidectine se caractérisait par un processus d'absorption plus rapide et une rémanence plus longue (48). Cette différence est la résultante d'une formulation différente (aqueuse) pour la moxidectine et d'un stockage plus intense au niveau graisseux.

Cet effet de la formulation sur le processus d'absorption a été longuement documenté tant pour l'ivermectine (51) que pour la doramectine (73). Cela a justifié le choix d'une formulation huileuse par Pfizer (doramectine) et Merck (ivermectine), tandis que Fort-Dodge (moxidectine), en raison du caractère lipophile plus marqué de la moxidectine, retenait un excipient aqueux afin de ne pas majorer l'importante rémanence résultant du stockage au niveau de la graisse.

\section{Influence de la voie d'administration}

La voie d'administration est un déterminant majeur à prendre en compte dans la recherche d'une efficacité optimale des endectocides. Dans le cas de l'ivermectine, une comparaison des différentes voies d'administration chez les bovins permet d'illustrer l'importance du mode de traitement (tableau IV). Les niveaux d'exposition engendrés par les différents types de traitement sont directement liés à la quantité d'ivermectine utilisée mais ne sont pas proportionnels. En effet, la mise en jeu du principe actif 2,5 fois supérieur dans le cas du pouron n'engendre pas d'exposition supérieure (mais inférieure) à celle générée par une administration par voie sous-cutanée (6).

Par ailleurs, l'usage de la voie pour-on génère plusieurs biais inhérents à ce type d'application : l'absorption percutanée est faible (biodisponibilité de $19 \pm 5$ p. 100) (47) ; le léchage des animaux eux-mêmes ou entre eux engendre une absorption du principe actif par voie orale qui augmente largement la biodisponibilité de la formulation (biodisponibilité de $33 \pm 18$ p. 100) mais qui est aussi à l'origine d'une très grande variabilité (47).

Dès lors, on peut considérer que la voie pour-on n'est pas une voie aussi performante qu'une administration sous-cutanée en termes de retour sur la dose mise en jeu. Elle présente le risque d'engendrer des niveaux de concentration subthérapeutiques chez de nombreux animaux traités (28) et des résidus inattendus chez des animaux non traités.

Dans le cas de la moxidectine, une comparaison des voies d'administration sous-cutanée et orale chez le mouton montre une équivalence en termes d'exposition révélée par la similitude des AUC $(98,89 \mathrm{ng} / \mathrm{j} / \mathrm{ml}$ pour la voie orale et $112,33 \mathrm{ng} / \mathrm{j} / \mathrm{ml}$ pour la voie sous-cutanée) (6). Néanmoins, pendant la période de 5 à 33 jours après l'administration, la voie sous-cutanée permet le maintien d'une concentration plasmatique de moxidectine qui est le double de celle obtenue par la voie orale (figure 4). Cette différence de rémanence est bien corrélée par les observations des parasitologues qui décrivent une persistance d'activité plus longue pour la voie sous-cutanée $(21,44)$.

L'ensemble des éléments précédemment rapportés montrent la prévalence de la voie d'administration sous-cutanée sur les autres voies d'administration (orale et pour-on); il s'agit d'un argument pharmacologique majeur qui mérite d'être pris en compte par les thérapeutes.

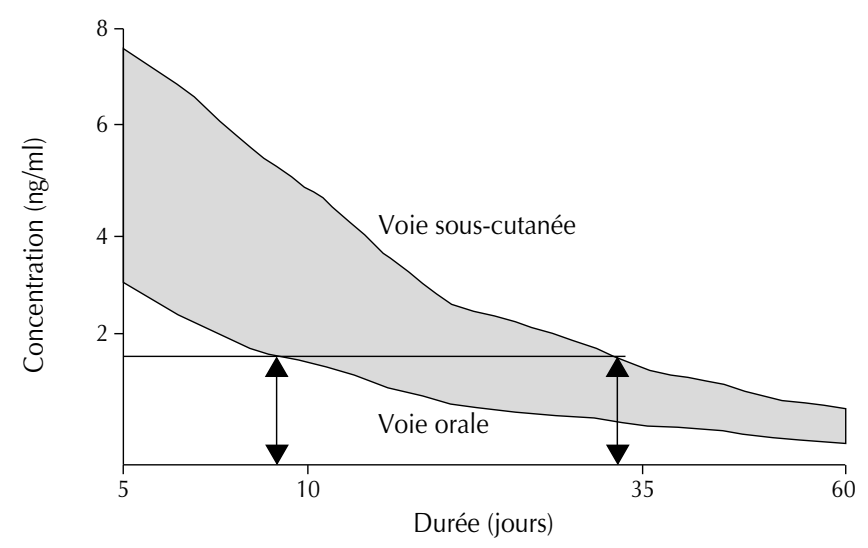

Figure 4 : comparaison entre la voie sous-cutanée et la voie orale (Alvinerie, 1998, Vet. Res.).

\section{Différences entre espèces animales cibles}

Des études comparées des niveaux d'exposition et de rémanence ont été réalisées chez de nombreuses espèces (vache, mouton, chèvre, cheval) ; elles montrent la grande variabilité de ces paramètres chez les différentes espèces $(5,36)$. A titre d'exemple, l'exposition à la doramectine générée par une administration sous-cutanée $(0,2 \mathrm{mg} / \mathrm{kg})$ est respectivement trois et six fois inférieure chez le mouton et la chèvre par rapport à celle obtenue chez la vache (figure 5). Des variations similaires sont décrites pour l'ensemble des lactones macrocycliques et interrogent sur

\section{Tableau IV}

Effet de la voie d'administration de l'ivermectine chez les bovins

\begin{tabular}{|c|c|c|c|c|}
\hline \multirow[t]{2}{*}{ Administration } & \multicolumn{3}{|c|}{ Paramètres } & \multirow[t]{2}{*}{ Référence } \\
\hline & $\begin{array}{c}\text { AUC } \\
\text { (ng/jour/ml) }\end{array}$ & $\underset{(\mathrm{ng} / \mathrm{ml})}{\mathrm{C}_{\max }}$ & $\begin{array}{l}\text { Biodisp. rel. - } \\
\operatorname{Vsc}^{1}(\%)\end{array}$ & \\
\hline $\begin{array}{l}\text { Sous-cutanée } \\
(0,2 \mathrm{mg} / \mathrm{kg})\end{array}$ & 459 & 42,8 & 100 & Lanusse et coll., 1997, J. vet. Pharmacol. Ther. \\
\hline $\begin{array}{l}\text { Pour-on } \\
(0,5 \mathrm{mg} / \mathrm{kg})\end{array}$ & 168 & 12,2 & 14,6 & Gayrard et coll., 1999, Vet. Parasitol. \\
\hline $\begin{array}{l}\text { Orale } \\
(8,6 \mathrm{mg} / \mathrm{kg})\end{array}$ & 2476 & 28,5 & 12,5 & Alvinerie et coll., 1998, Vet. Res. \\
\hline
\end{tabular}

${ }^{1}$ Biodisponibilité relative par rapport à la voie sous-cutanée 


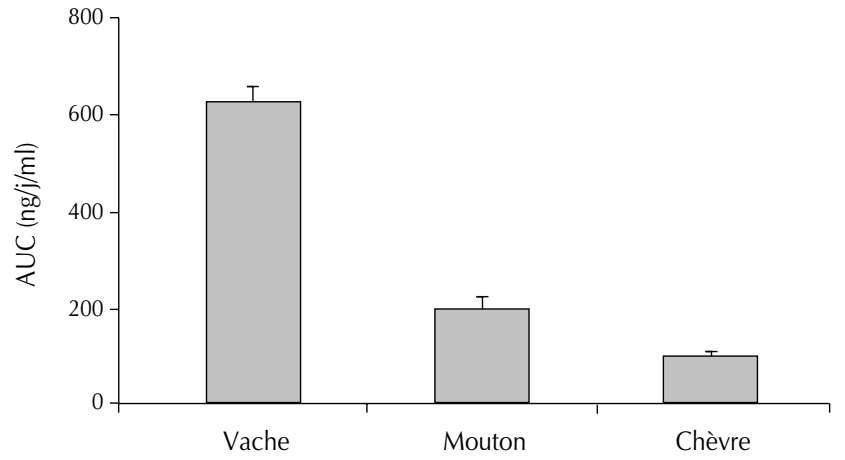

Figure 5 : exposition à la doramectine générée par une administration sous-cutanée de $0,2 \mathrm{mg} / \mathrm{kg}$ chez trois espèces de ruminants (Barber et coll., 2003, J. vet. Pharmacol. Ther. ; Escudero et coll., 1998, Res. vet. Sci. ; Lanusse et coll., 1997, J. vet. Pharmacol. Ther.).

la pertinence de la transposition de la dose sélectionnée chez la vache aux autres espèces.

L'exposition dramatiquement faible observée chez la chèvre après administration sous-cutanée et surtout orale (5) pourrait être corrélée à l'émergence plus précoce de parasites résistants aux endectocides chez cette espèce. Cet exemple illustre parfaitement tout le danger des concentrations subthérapeutiques.

Des travaux plus récents conduits expérimentalement sur l'usage de l'éprinomectine chez la chèvre ont montré l'efficacité suboptimale de la formulation pour-on à la dose de $0,5 \mathrm{mg} / \mathrm{kg}$, tandis qu'une dose double $(1 \mathrm{mg} / \mathrm{kg})$ satisfait tant aux exigences d'efficacité qu'à celles de la sécurité de la production laitière (22). L'ensemble de ces éléments devrait conduire la firme à déposer un dossier d'enregistrement pour l'usage de l'éprinomectine chez la chèvre avec une dose spécifique à cette espèce.

\section{RECOMMANDATIONS D'USAGE PROPRES} AU CONTEXTE AFRICAIN

Les données publiées sur l'efficacité et la pharmacologie des antiparasitaires endectocides dans le contexte vétérinaire africain sont rares bien que ces molécules soient déjà largement utilisées par les éleveurs et les vétérinaires dans de nombreux pays d'Afrique de l'Ouest et du Centre. Une adaptation des schémas thérapeutiques tenant compte des particularités d'ordre physiologique et pharmacologique des différentes races et des contraintes environnementales est nécessaire étant donné l'importance de cette classe thérapeutique dans la lutte contre les parasites du bétail. Les facteurs à prendre en compte dans cette stratégie pouvant faire l'objet d'une évaluation pharmacocinétique sont multiples ; l'accent doit être mis sur quelques uns d'entre eux.

\section{Particularité de l'infestation parasitaire dans le contexte africain}

L'appréciation de l'effet du parasitisme et notamment d'une infection par des nématodes gastro-intestinaux sur la productivité du bétail et le développement de mesures de lutte appropriées et durables nécessitent une bonne connaissance de leur inventaire, leur épidémiologie et leurs effets pathologiques. Les régions intertropicales d'Afrique présentent une gamme très étendue de milieux: forêts denses équatoriales, savanes arborées, steppes sahéliennes, grandes étendues dunaires des zones sahariennes. De nombreux parasites sont présents dans ces milieux et ont développé des adaptations très performantes pour leur survie et leur maintien dans des zones aussi déshéritées que le Sahel ou le Sahara $(3,17$, 19, 41, 46). La zone tropicale humide offre des conditions favorables à l'évolution des stades libres pendant toute l'année. La zone soudano-sahélienne, en revanche, ne permet plus cette évolution que quatre ou cinq mois par an. Cette période favorable est réduite à deux mois en zone sahélo-sahélienne (32).

Des études épidémiologiques, bien que peu abondantes, ont révélé que la quasi-totalité des ruminants sont porteurs de nématodes gastro-intestinaux et d'ectoparasites. Les espèces prédominantes sont Haemonchus sp., Cooperia spp., Oesophagostomum radiatum et Bunostomum phlebotomum $(16,52,55,69,70)$. La charge parasitaire suit une évolution saisonnière et 80 p. 100 de cette charge est observée pendant la saison des pluies. Les réinfections sont négligeables pendant la saison sèche en zone sahélienne $(8,78)$. Haemonchus sp. survit presque exclusivement en tant que larve hypobiotique (stade inhibé dans la muqueuse de la caillette) pendant la longue saison sèche en zone sahélo-sahélienne (40, 41, 43, 53, 54). En revanche, Cooperia spp. et $O$. radiatum survivent partiellement en tant qu'adultes hypométaboliques avec une fécondité réduite et partiellement en tant que larves dans la muqueuse intestinale (43, 52, 55). La contamination des pâturages recommence avant le début de la saison des pluies surtout par la réactivation des larves hypobiotiques (43).

\section{Particularités physiologiques des zébus africains}

La physiologie de l'organisme animal hôte conditionne très largement le devenir du médicament, sa concentration au niveau de la cible parasitaire et, in fine, son efficacité. Des travaux en cours ont permis de mettre en évidence des différences importantes en terme de biodisponibilité des endectocides entre le zébu Gobra du Sénégal et les autres bovins (figure 6). L'administration de l'ivermectine à la dose de $0,2 \mathrm{mg} / \mathrm{kg}$ génère chez le zébu Gobra une AUC 2,3 fois plus faible que celle obtenue dans les mêmes conditions chez les taurins. De même, l'administration de l'éprinomectine par la voie percutanée à la dose de $0,5 \mathrm{mg} / \mathrm{kg}$ se traduit par une disponibilité encore plus faible et par de très grosses variations individuelles. Ces différences sont vraisemblablement la conséquence de différences physiologiques et métaboliques entre le zébu Gobra et les autres bovins. Malgré un manque général de performances, les races africaines sont bien adaptées pour survivre et se reproduire dans leur environnement. En effet, elles sont bien connues pour leur résistance et leur tolérance aux différents stress environnementaux : sous-alimentation, chaleurs, humidité, maladies $(4,30,31,58)$. Les contraintes alimentaires sont à l'origine d'un niveau d'engraissement faible ;

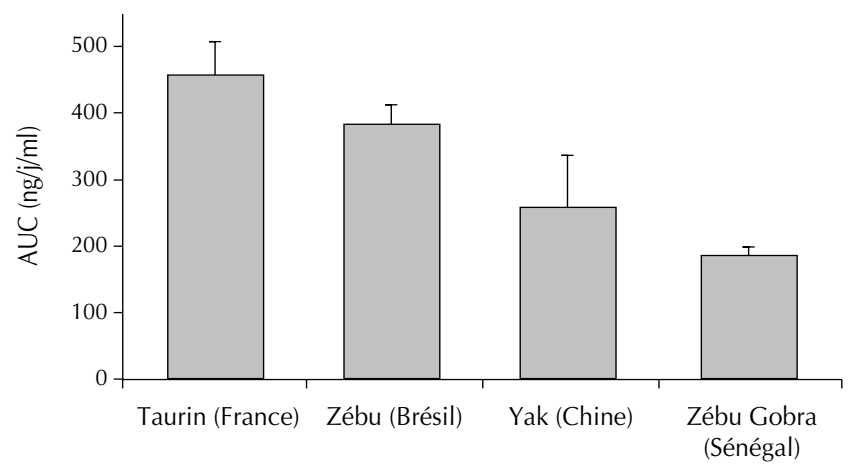

Figure 6 : exposition de différents bovidés à l'ivermectine après administration sous-cutanée à la dose de $0,2 \mathrm{mg} / \mathrm{kg}$ (Bengone et coll., 2004, Vet. Parasitol. (in press) ; Dupuy et coll., 2003, Vet. Parasitol. ; Lanusse et coll., 1997, J. vet. Pharmacol. Ther.). 
elles pourraient expliquer les différences observées au plan pharmacocinétique entre le zébu Gobra et les autres bovins, qui sont de nature à affecter les propriétés pharmacologiques des endectocides. Des études complémentaires sont sans aucun doute nécessaires pour préciser l'efficacité des différentes doses d'endectocides sur les nématodes et les ectoparasites dans le contexte africain afin d'envisager une adaptation posologique.

\section{Formulations et problématique des génériques sur le marché africain}

L'importance de la formulation des endectocides a été discutée dans la deuxième partie de cette synthèse. Il convient cependant de souligner son intérêt particulier dans le contexte africain avec notamment l'usage assez répandu des formulations génériques et la circulation des médicaments issus de la contrefaçon. Les contrefaçons et les malfaçons des médicaments sont un fait courant dans les pays en développement. Ce phénomène a été décrit en Afrique de l'Ouest aussi bien pour les médicaments humains que pour les médicaments vétérinaires (67). Il est probablement lié à des systèmes d'approvisionnement et de distribution mal réglementés.

Les actions de sensibilisation sur le bon usage des médicaments vétérinaires sont cependant en cours dans certains de ces pays ; le séminaire sur la qualité des médicaments vétérinaires en Afrique francophone tenue à Dakar en décembre 1999 a adopté un certain nombre de recommandations visant à pallier les carences existant en matière de contrôle des médicaments vétérinaires (2). Les travaux réalisés au sein du laboratoire de contrôle des médicaments vétérinaires de l'Ecole inter-Etats des sciences et médecine vétérinaires (Eismv) de Dakar doivent être encouragés. Dans le cas particulier des endectocides, il est impératif que ces travaux assurent à terme la distribution de produits de qualité dont la bioéquivalence aura été montrée par des études appropriées de biodisponibilité.

\section{- CONCLUSION}

L'arsenal antiparasitaire a évolué, plus particulièrement dans les années 1980 avec l'apparition des macrolides endectocides. Les cinq molécules actuellement sur le marché présentent un intérêt majeur dans la lutte contre les parasites externes et internes des animaux. La chimiothérapie est, et restera sans doute longtemps, la pierre angulaire de la stratégie antiparasitaire, surtout en l'absence de méthodes alternatives. Le risque de défaillance des endectocides dans l'avenir prendra pour origine un emploi excessif et/ou un choix erroné dans les doses ou les voies d'administration utilisées. L'utilisation optimale du traitement anthelminthique dans une démarche de gestion du risque parasitaire dans les élevages africains nécessite :

- de développer une bonne connaissance des parasites dans chaque milieu ;

- de connaître en tout temps l'état de l'infestation et d'évaluer le niveau du risque ;

- de mettre en place des mesures préventives ;

- de disposer de plusieurs moyens curatifs ;

- d'évaluer l'efficacité des différents antiparasitaires afin de chercher l'harmonie et l'efficacité des interventions ;

- d'être en mesure de vérifier les impacts et l'efficacité des mesures ; - d'adapter les interventions curatives et la stratégie de lutte en fonction de la situation.

Il s'agit d'un véritable défi pour l'avenir de la thérapeutique antiparasitaire et le développement de l'élevage dans les pays africains ; il doit résulter d'une meilleure coopération entre les différents partenaires impliqués dans la mise en œuvre des traitements.

\section{Remerciements}

Ce travail a bénéficié du soutien de l'Agence universitaire de la francophonie (AUF), bureau Afrique de l'Ouest. Les auteurs remercient particulièrement $\mathrm{M}$. Bonaventure Mvé Ondo pour ses encouragements, ainsi que le Pr Josef Vercruysse pour ses conseils pertinents.

\section{BIBLIOGRAPHIE}

1. ABBOTT K.A., COBB R.M., GLASS M.H., 1995. Duration of the persistent activity of moxidectin against Haemonchus contortus in sheep. Aust. vet. J., 72: 408-410.

2. ABIOLA F.A., 2000. Un laboratoire de contrôle des médicaments vétérinaires à l'Eismv de Dakar. In : Actes $4^{\mathrm{e}}$ Séminaire sur les médicaments vétérinaires en Afrique, Dakar, Sénégal, 6-10 décembre 1999. Paris, France, OIE.

3. ACHI Y.L., ZINSSTAG J., YAO K., YEO N., DORCHIES P., JACQUIET P., 2003. Host specificity of Haemonchus spp. for domestic ruminants in the savanna in northern Ivory Coast. Vet. Parasitol., 116: 151-158.

4. AKINBAMIJO O.O., BENNISON J.J., ROMNEY D.L., WASSINK G.J., JAITNER J., CLIFFORD D.J., DEMPFLE L., 1997. An evaluation of food intake, digestive, physiology and live-weight changes in $\mathrm{N}^{\prime}$ dama and Gobra zebu bulls following experimental Trypanosoma congolense infection. Anim. Sci., 65: 151-158.

5. ALVINERIE M., 1997. Comparative pharmacokinetic properties of ivermectin and moxidectin in different animal species. J. vet. Pharmacol. Ther., 20: 74-76.

6. ALVINERIE M., ESCUDERO E., SUTRA J.F., GALTIER P., 1998. The pharmacokinetics of moxidectin after oral and subcutaneous administration to sheep. Vet. Res., 29: 113-118.

7. ALVINERIE M., SUTRA J.F., GALTIER P., MAGE C., 1999. Pharmacokinetics of eprinomectin in plasma and milk following topical administration to lactating dairy cattle. Res. vet. Sci., 67: 229-232.

8. ANKERS P., ZINSSTAG J., PFISTER K., 1994. Quasi-absence de réinfestation par les strongles du bétail gambien en saison sèche. Revue Elev. Méd. vét. Pays trop., 47 : 201-205.

9. ARENA J.P., LIU K.K., PARESS P.S., FRAZIER E.G., CULLY D.F., MROZIK H., SCHAEFFER J.M., 1995. The mechanism of action of avermectins in Caenorhabditis elegans: correlation between activation of glutamatesensitive chloride current, membrane binding, and biological activity. J. Parasitol., 81: 286-294.

10. BARBER S., BOWLES V., LESPINE A., ALVINERIE M., 2003. The comparative serum disposition kinetics of subcutaneous administration of doramectin, ivermectin and moxidectin in the Australian Merino sheep. J. vet. Pharmacol. Ther., 26: 343-348.

11. BENGONE NDONG T., BA M.A., KANE Y., SANE I., SUTRA J.F., ALVINERIE M., 2004. Pharmacokinetics of ivermectin in zebu Gobra (Bos indicus). Vet. Parasitol. (in press)

12. BISSET S.A., VLASSOF A., MCMURTRY L.W., ELLIOTT D.C., COBB R.M., KIERAN P.J., WOOD I.B., 1992. An evaluation of an oral administration of moxidectin against selected anthelmintic-resistant and susceptible strains of nematodes in lambs. N. Z. vet. J., 40: 97-100.

13. BLOOMQUIST J., 2003. Chloride channels as tools for developing selective insecticides. Arch. Insect. Biochem. Physiol., 54: 145-156.

14. CARTER G.T., NIETSCHE J.A., BORDERS D.B., 1987. Structure determination of LL-F28249 $\alpha, \beta, \gamma$ and $\lambda$, potent antiparasitic macrolides from Streptomyces cyaneogriseus spp. noncyanogenus. J. chem. Soc. Chem. Commun., 6: 402-404.

15. CHABALA J.C., MROZIK H., TOLMAN R.L., ESKOLA P., LUSI A., PETERSON L.H., WOODS M.F., FISHER M.H., 1980. Ivermectin, a new broad-spectrum antiparasitic agent. J. Med. Chem., 23: 1134-1136.

16. CHARTIER C., BUSHU M., KAMWENGA D., 1991. Les dominantes du parasitisme helminthique chez les bovins en Ituri (Haut-Zaïre). III. Répartition géographique et prévalence des principaux helminthes. Revue Elev. Méd. vét. Pays trop., $44: 61-68$

17. CHOLLET J.Y., JACQUIET P., CARDINALE E., NDAMKOU-NDAMKOU C., DIOP C., THIAM A., DORCHIES P., 2000. Cooperia pectinata and C. punctata, parasites of the abomasum of cattle in northern Cameroon (Central Africa). Vet. Parasitol., 88: 135-138. 
18. COURTNEY C.H., SHEARER J.K., PLUE R.E., 1985. Efficacy and safety of clorsulon used concurrently with ivermectin for control of Fasciola hepatica in Florida USA beef cattle. Am. J. vet. Res., 46: 1245-1246.

19. DIA M.L., JACQUIET P., CHOLLET J.Y., DIOP C., THIAM A., AMINETOU M., AHMED SALEM C.B., MREZIG A., DIALLO B.C., 2000. Ten years of research activities on tropical parasitology of dromedaries, cattle, and small ruminants in Mauritania. Ann. N. Y. Acad. Sci., 916: 404-409.

20. DOBSON R.J., BESIER R.B., BARNES E.H., LOVE S.C., VIZARD A., BELL K., LE JAMBRE L.F., 2001. Principles for the use of macrocyclic lactones to minimise selection for resistance. Aust. vet. J., 79: 756-761.

21. DORCHIES P., CARDINAUD B., FOURNIER R., 1996. The persistence of efficacy of moxidectin as a $1 \%$ injectable solution and a $0.1 \%$ oral drench against nasal bots, pulmonary and gastro-intestinal nematodes in sheep. Vet. Parasitol., 65: 163-168.

22. DUPUY I., CHARTIER C., SUTRA J.F., ALVINERIE M., 2001. Eprinomectin in dairy goats: dose influence on plasma and excretion in milk. Parasitol. Res., 87: 294-298.

23. DUPUY J., YIN H., LUO J.X., YANG D.Y., GUAN G.Q., MA M.L., SUTRA J.F., LESPINE A., BOULARD C., ALVINERIE M., 2003. Pharmacokinetics of ivermectin in the yak (Bos grunniens). Vet. Parasitol., 117: 153-157.

24. EGERTON J.R., OSTLIND D.A., BLAIR L.S., EARY C.H., SUHAYDA D., CIFELLI S., RIEK R.F., CAMPBELL W.C., 1979. Avermectins, new family of potent anthelmintic agents: efficacy of the $\mathrm{B}_{12}$ component. Antimicrob. Agents Chemother., 15: 372-378

25. EGERTON J.R., BIRNBAUM J., BLAIR L.S., CHABALA J.C., CONROY J., FISHER M.H., MROZIK H., OSTLIND D.A., WILKINS C.A., CAMPBELL W.C., 1980. 22,23-dihydroavermectin $B_{1}$, a new broad-spectrum antiparasitic agent. Br. vet. J., 136: 88-97.

26. ESCUDERO E., CARCELES C.M., DIAZ M.S., SUTRA J.F., GALTIER P., ALVINERIE M., 1998. Pharmacokinetics of mixodectin and doramectin in goats. Res. vet. Sci., 67: 177-181.

27. FELLOWES R.A., MAULE A.G., MARTIN R.J., GEARY T.G., THOMPSON D.P., KIMBER M.J., MARKS N.J., HALTON D.W., 2000. Classical neurotransmitters in the ovijector of Ascaris suum: localization and modulation of muscle activity. Parasitology, 121: 325-336.

28. GAYRARD V., ALVINERIE M., TOUTAIN P.L., 1999. Comparison of pharmacokinetic profiles of doramectin and ivermectin pour-on formulations in cattle. Vet. Parasitol., 81: 47-55.

29. GOUDIE A.C., EVANS N.A., GRATION K.A.F., BISHOP B.F., GIBSON S.P., HOLDOM K.S., KAYE B., WICKS S.R., LEWIS D., WEATHERLEY A.J., BRUCE C.I., HERBERT A., SEYMOUR D.J., 1993. Doramectin - a potent novel endectocide. Vet. Parasitol., 49: 5-15.

30. GRIMAUD P., RICHARD D., KANWE A., DURIER C., DOREAU M. 1998. Effect of undernutrition and refeeding on digestion in Bos taurus and Bos indicus in a tropical environment. Anim. Sci., 67: 49-58.

31. GRIMAUD P., RICHARD D., VERGERON M.P., GUILLERET J.R., DOREAU M., 1999. Effect of drastic undernutrition on digestion in Zebu cattle receiving a diet based on rice straw. J. Dairy Sci., 82: 974-981.

32. GUEYE A., MBENGUE M., DIOUF A., 1994. Tiques et hémoparasitoses du bétail au Sénégal. VI. La zone soudano-sahélienne. Revue Elev. Méd. vét. Pays trop. $47:$ 39-46.

33. HEINZE-MUTZ E.M., PITT S.R., BAIRDEN K., BAGGOT D.G., ARMOUR J., BARTH D., CRAMER L.G., 1993. Efficacy of abamectin against nematodes in cattle. Vet. Rec., 132: 35-37.

34. HEINZE-MUTZ E.M., BARTH D., CRAMER L.G., CROSS S.J., VISSER M., 1993. Efficacy of abamectin against ectoparasites of cattle. Vet. Rec., 132: $455-457$

35. HEJMADI M.V., JAGANNATHAN S., DELANY N.S., COLES G.C., WOLSTENHOLME A.J., 2000. L-glutamate binding sites of parasitic nematodes: an association with ivermectin resistance? Parasitology, 120: 535-545.

36. HENNESSY D.R., ALVINERIE M., 2002. Pharmacokinetics of the macrocyclic lactones: conventional wisdom and new paradigms. In: Vercruysse J., Rew R.S. Eds, Macrocyclic lactones in antiparasitic therapy. Cambridge, MA, USA, CABI Publishing, p. 97-123.

37. HOTSON I.K., 1982. The avermectins: a new family of antiparasitic agents. J. S. Afr. Vet. Assoc., 53: 87-90.
38. IKEDA T., 2003. Pharmacological effects of ivermectin, an antiparasitic agent for intestinal strongyloidiasis: its mode of action and clinical efficacy. Folia Pharmacol. Jpn, 122: 527-538.

39. JACKSON H.C., 1989. Ivermectin as a systemic insecticide. Parasitol. Today, 5: 146-156.

40. JACQUIET P., HUMBERT J.F., COMES A.M., CABARET J., THIAM E., CHEIKH D., 1995. Ecological, morphological and genetic characterization of sympatic Haemonchus spp. parasites of domestic ruminants in Mauritania. Parasitology, 110: 483-492.

41. JACQUIET P., CABARET J., THIAM E., CHEIKH D., 1998. Experimental and natural Haemonchus spp. cross infections of domestic ruminants in Sahelian West Africa. Ann. N. Y. Acad. Sci., 849: 465-469.

42. JOHNSON E.G., 1991. Effects of liver flukes on feedlot performance. Agri-Pract., 12: 33-34

43. KAUFMANN J., PFISTER K., 1990. The seasonal of gastrointestinal nematodes in N'Dama cattle in the Gambia. Vet. Parasitol., 43: 157-170.

44. KERBOEUF D., HUBERT J., CARDINAUD B., BLOND-RIOU F., 1995. The persistence of efficacy of injectable or oral moxidectin in experimentally injected sheep. Vet. Rec., 137: 399-401.

45. KOHLER P., 2001. The biochemical basis of anthelmintic action and resistance. Int. J. Parasitol., 31: 336-345.

46. KOMOIN-OKA C., ZINSSTAG J., PANDEY V.S., FOFANA F., N'DEPO A., 1999. Epidémiologie des parasites des ovins de la zone sud forestière de la Côte d'Ivoire. Revue Elev. Méd. vét. Pays trop., 52 : 39-46.

47. LAFONT C.M., ALVINERIE M., BOUSQUET-MELOU A., TOUTAIN P.L., 2001. Licking behaviour and environmental contamination arising from pour-on ivermectin for cattle. Int. J. Parasitol., 31: 1687-1692.

48. LANUSSE C. LIFSCHITZ A., VIRKEL G, ALVAREZ L., SANCHEZ S, SUTRA J.F., ALVINERIE M., 1997. Comparative plasma disposition kinetics of ivermectin, moxidectin and doramectin in cattle. J. vet. Pharmacol. Ther., 20: 91-99.

49. LIFSCHITZ A., VIRKEL G., IMPERIALE F., GALTIER P., LANUSSE C., ALVINERIE M., 1999. Moxidectin in cattle: correlation between plasma and target tissues disposition. J. vet. Pharmacol. Ther., 22: 266-273.

50. LIFSCHITZ A., VIRKEL G., SALLOVITZ J., SUTRA J.F., GALTIER P ALVINERIE M., LANUSSE C., 2000. Comparative distribution of ivermectin and doramectin to parasite location tissues in cattle. Vet. Parasitol., $\mathbf{8 7}$ 327-338.

51. LO P.K., FINK D.W., WILLIAMS J.B., BLODINGER J., 1985 Pharmacokinetic studies of ivermectin: effect of formulation. Vet. Res. Commun., 9: 251-268.

52. NDAO M., BELOT J., ZINSSTAG J., PFISTER K., 1995. Epidémiologie des nématodes gastro-intestinaux des ruminants dans la zone syvopastorale au Sénégal. Vet. Res., 26 : 132-139.

53. NDAO M., PANDEY V.S., ZINSSTAG J., PFISTER K., 1995. Helminth parasites and hypobiosis of nematodes in N'Dama cattle during the dry season in the Gambia. Vet. Parasitol., 60: 161-166.

54. NDAO M., PANDEY V.S., ZINSSTAG J., PFISTER K., 1995. Effect of a single dry season anthelmintic treatment of $\mathrm{N}^{\prime}$ Dama cattle on communal pastures in the Gambia. Vet. Res. Commun., 19: 205-213.

55. NDAO M., BELOT J., ZINSSTAG J., PFISTER K., 1995. Epidémiologie des nématodes gastro-intestinaux des bovins dans la zone syvo-pastorale au Sénégal. Vet. Res., 146 : 129-134.

56. PITT S.R., LANGHOLFF W.K., EAGLESON J.S., REHBEIN S., 1997. The efficacy of eprinomectin against induced infections of immature (fourth larval stage) and adult nematode parasites in cattle. Vet. Parasitol., 73 119-128.

57. RANJAN S., TRUDEAU C., PRICHARD R.K., VON KUTZLEBEN R., CARRIER R., 1992. Efficacy of moxidectin against naturally acquired nematode infections in cattle. Vet Parasitol, 41:227-241.

58. RICHARD D., GUERIN H., FALL S.T., 1989. Feeds in the dry tropics. In: Jarrige R. Ed., Ruminant nutrition. Recommended allowance and feed tables. Paris, France, INRA, p. 345-357.

59. ROBIN B., 1983. Ivermectine : 22, 23 dihydroavermectine $B_{1}$ : un nouvel antiparasitaire à très large spectre. Revue Méd. vét., 134 : 495-498.

60. ROULET A., PUEL O., GESTA S., LEPAGE J.F., DRAG M., SOLL M. ALVINERIE M., PINEAU T., 2003. MDR1-deficient genotype in Collie dogs hypersensitive to the P-glycoprotein substrate ivermectin. Eur. J. Phamacol., 460: $85-91$. 
61. SCHINKEL A, WAGENAAR E, MOL C , DEEMPTER L.V., 1996. P glycoprotein in the blood-brain barrier of mice affects brain penetration and pharmacological activity of many drugs. J. clin. Invest., 97: 2517-2524.

62. SHOOP W.L., MROZIK H., FISHER M.H., 1995. Structure and activity of avermectins and milbemycins in animal health. Vet. Parasitol., 59: 139-156.

63. SHOOP W.L., OSTLIND D.A., ROHRER S.P., MICKLE G., HAINES H.W., MICHAEL B.F., MROZIK H., FISHER M.H., 1995. Avermectins and milbemycins against Fasciola hepatica: in vivo drug efficacy and in vitro receptor binding. J. int. Parasitol., 25: 923-927.

64. SHOOP W.L., DEMONTIGNY P., FINK D.W., 1996. Selection of a novel avermectin for use as a topical endectocide for cattle. Int. J. Parasitol., 26: 1227-1235.

65. SHOOP W.L., EGERTON J.R., EARY C.H., HAINES H.W., MICHAEL B.F., MROZIK H., ESKOLA P., FISHER M.H., SLAYTON L., OSTLIN D.A., SKELLY B.J., FULTON R.K., BARTH D., COSTA S., GREGORY L.M. CAMPBELL W.C., SECARD R.L., TURNER M.J., 1996. Eprinomectin: a novel avermectin for use as a topical endectocide for cattle. Int. J. Parasitol., 26: 1237-1242.

66. TAKIGUCHI Y., MISHIMA H., OKUDA M., TERAO M., 1980. Milbemycins, a new family of macrolide antibiotics: fermentation, isolation and physico-chemical properties. J. Antibiot., 33: 1120-1127.

67. TEKO-AGBO A., BIAOU C., AKODA K., FAURE P., ABIOLA F.A. 2003. Contrefaçons et malfaçons des trypanocides à base de diminazène et d'anthelminthiques contenant de I'albendazole au Bénin et au Togo. RASPA, 1 : 39-47.

68. TURNER M., SHAEFFER J., 1989. Mode of action of ivermectin. In: Campbell W. Ed., Ivermectin and abamectin. New York, USA, SpringerVerlag, p. 73-88.

69. VASSILIADES G., 1978. Les affections parasitaires dues à des helminthes chez les bovins du Sénégal. Revue Elev. Méd. vét. Pays trop., 31 : 157-163.

70. VASSILIADES G., 1984. Parasitisme gastro-intestinal chez le mouton du Sénégal. Revue Elev. Méd. vét. Pays trop., 34 : 169-177.

\section{Summary}

Bengone-Ndong T., Alvinerie M. Antiparasitic Macrolides: General Pharmacological Properties and Usage Instructions in the African Veterinary Context

Antiparasitic macrolides (or endectocides) represent the most recent class of antiparasitic drugs. This class is homogeneous because of its unique and specific, glutaminergic, mode of action, and its pharmacological properties. These drugs have a spectrum of action directed against many nematodes, as well as many insects and mites. Their structures and associated galenic formulations also enable them to have a high remanent potential. Unfortunately, endectocides are generally used in African countries without any recording procedure. Moreover, the introduction of generic formulations facilitated massive and repetitive uses that have led to therapeutic failures. These drugs should be given a good set-up to be effective in an African tropical environment, in particular by taking into account the characteristics of local breeds and environmental constraints. The first element to consider is the choice of the most potent route of administration. At the same time, the differences observed between animal species targets as well as physiological peculiarities must lead to a better adapted use.

Key words: Parasitism - Veterinary drugs - Medical properties Efficiency - Africa.
71. VERCRUYSSE J., CLAEREBOUT E., DORNY P., DEMEULENAERE D., AGNEESSENS J., SMETS K., 1998. Persistence of the efficacy of doramectin against Ostertagia ostertagi and Cooperia oncophora in cattle. Vet. Rec., 143: 443-446.

72. WARDHAUGH K.G., MAHON R.J., AHMAD H.B., 2001. Efficacy of macrocyclic lactones for the control of larvae of the Old World screwworm fly (Chrysomya bezziana). Aust. vet. J., 79: 120-124.

73. WICKS S.R., KAYE B., WEATHERLEY A.J., SMITH D.G., 1993. Effect of formulation on the pharmacokinetics and efficacy of doramectin. Vet. Parasitol., 49: 17-26.

74. WILLIAMS J.C., BROUSSARD S.D., 1995. Persistent anthelmintic activity of ivermectin against gastrointestinal nematodes of cattle. Am. J. vet. Res., 56: 1169-1175.

75. WILLIAMS J.C., STUEDEMANN J.A., BAIRDEN K., KERBOEUF D., GIODIA H., HUBERT J., BROUSSARD S.D., PLUE R.E., ALVA-VALDES R., BAGGOTT D.G., PINKALL N., EAGLESON J.S., 1997. Efficacy of a pour-on administration of eprinomectin (MK-397) against nematode parasites of cattle with emphasis on inhibited early fourth-stage larvae of Ostertagia spp. Am. J. vet. Res., 58: 379-383.

76. WILLIAMS J.C., LOYACANO A.F., DEROSA A., GURIE J., CLYMER B.C., GUERINO F., 1999. A comparison of persistent anthelmintic efficacy of topical formulations of doramectin, ivermectin, eprinomectin and moxidectin against naturally acquired nematode infections of beef calves. Vet. Parasitol., 85: 277-288.

77. ZHANG M., WANG G., SHAPIRO A., ZHANG J., 1996. Topological folding and proteolysis profile of P-glycoprotein in membranes of multidrug-resistant cells: implication for the drug-transport mechanism. Biochemistry, 35: 9728-9736.

78. ZINSSTAG J., NIIE M., KAUFMANN J., PFISTER K., 1994. Prolonged suppression of trichostrongyle egg output of N'Dama cattle by a single larvicidal treatment. Acta trop., 58: 99-103.

Reçu le 24.08.2004, accepté le 19.11.2004

\section{Resumen}

Bengone-Ndong T., Alvinerie M. Macrólidos antiparasitarios: propiedades farmacológicas generales y recomendaciones de uso en el contexto veterinario africano

Los macrólidos antiparasitarios, o endectocidas, representan la clase de antiparasitarios más reciente. Esta clase es homogénea debido a su modo de acción único y especifico, glutaminérgico, y a sus propiedades farmacológicas. Estas substancias tienen un espectro de actividad que se extiende a numerosos nemátodos, así como a numerosos insectos y ácaros, debido a su estructura y formulación galénica asociadas, representan igualmente una importante remanencia. Los endectocidas son desgraciadamente a menudo comercializados en el mercado africano sin ningún procedimiento de registro. Aún más, la introducción de las fórmulas genéricas ha facilitado el uso masivo y repetitivo, susceptible de contribuir a los fracasos terapéuticos. Conviene dar estos antiparasitarios en las mejores condiciones, para que ejerzan su acción en el medio tropical africano, teniendo en cuenta principalmente las particularidades de las razas locales y los inconvenientes del medio ambiente. Entre las medidas que se deben poner en marcha, la selección de la vía de administración mas eficaz constituye el primer elemento a considerar. Igualmente, las diferencias observadas entre las especies animales que se enfocan, así como las particularidades fisiológicas deben conducir a una utilización mejor adaptada.

Palabras clave: Parasitismo - Medicamento veterinario Propriedad medicinal - Eficacia - Africa. 